\title{
The PAMELA Experiment: A Cosmic Ray Experiment Deep Inside the Heliosphere
}

\author{
Mirko Boezio*1 $^{* 1}$ R. Munini ${ }^{1}$, O. Adriani ${ }^{2,3}$, G. C. Barbarino ${ }^{4,5}$, G. A. Bazilevskaya ${ }^{6}$, \\ R. Bellotti ${ }^{7,8}$, E. A. Bogomolov ${ }^{9}$, M. Bongi ${ }^{2,3}$, V. Bonvicini ${ }^{1}$, S. Bottai ${ }^{3}$, A. Bruno ${ }^{8}$, \\ F. Cafagna ${ }^{8}$, D. Campana ${ }^{5}$, P. Carlson ${ }^{10}$, M. Casolino ${ }^{11,12}$, G. Castellini ${ }^{13}$, \\ C. De Santis ${ }^{11}$, V. Di Felice ${ }^{11,14}$, A. M. Galper ${ }^{15}$, A. V. Karelin ${ }^{15}$, S. V. Koldashov ${ }^{15}$, \\ S. Koldobskiy ${ }^{15}$, S. Y. Krutkov ${ }^{9}$, A. N. Kvashnin ${ }^{6}$, A. Leonov ${ }^{15}$,V. Malakhov ${ }^{15}$, \\ L. Marcelli ${ }^{11}$, M. Martucci ${ }^{16,17}$, A. G. Mayorov ${ }^{15}$, W. Menn ${ }^{18}$, M. Mergé ${ }^{11,16}$, \\ V. V. Mikhailov ${ }^{15}$,E. Mocchiutti ${ }^{1}$, A. Monaco ${ }^{7,8}$, N. Mori $^{3}$, G. Osteria ${ }^{5}$, B. Panico ${ }^{5}$, \\ P. Papini ${ }^{3}$, M. Pearce ${ }^{10}$, P. Picozza ${ }^{11,16}$, M. Ricci ${ }^{17}$, S. B. Ricciarini ${ }^{13}$, M. Simon ${ }^{18}$, \\ R. Sparvoli ${ }^{11,16}$, P. Spillantini ${ }^{2,3}$, Y. I. Stozhkov ${ }^{6}$, A. Vacchi $^{1,19}$, E. Vannuccini ${ }^{3}$, \\ G. Vasilyev ${ }^{9}$, S. A. Voronov ${ }^{15}$, Y. T. Yurkin ${ }^{15}$, G. Zampa ${ }^{1}$, N. Zampa ${ }^{1}$ \\ ${ }^{1}$ INFN, Sezione di Trieste I-34149 Trieste, Italy \\ ${ }^{2}$ University of Florence, Department of Physics, I-50019 Sesto Fiorentino, Florence, Italy \\ ${ }^{3}$ INFN, Sezione di Florence, I-50019 Sesto Fiorentino, Florence, Italy \\ ${ }^{4}$ University of Naples "Federico II", Department of Physics, I-80126 Naples, Italy \\ ${ }^{5}$ INFN, Sezione di Naples, I-80126 Naples, Italy \\ ${ }^{6}$ Lebedev Physical Institute, RU-119991, Moscow, Russia \\ ${ }^{7}$ University of Bari, Department of Physics, I-70126 Bari, Italy \\ ${ }^{8}$ INFN, Sezione di Bari, I-70126 Bari, Italy \\ ${ }^{9}$ Ioffe Physical Technical Institute, RU-194021 St. Petersburg, Russia \\ ${ }^{10}$ KTH, Department of Physics, and the Oskar Klein Centre for Cosmoparticle Physics, AlbaNova \\ University Centre, SE-10691 Stockholm, Sweden \\ ${ }^{11}$ INFN, Sezione di Rome "Tor Vergata", I-00133 Rome, Italy \\ ${ }^{12}$ RIKEN, Advanced Science Institute, Wako-shi, Saitama, Japan \\ 13 IFAC, I-50019 Sesto Fiorentino, Florence, Italy \\ ${ }^{14}$ Agenzia Spaziale Italiana (ASI) Science Data Center, I-00044 Frascati, Italy \\ 15 National Research Nuclear University MEPhI, RU-115409 Moscow \\ 16 University of Rome "Tor Vergata”, Department of Physics, I-00133 Rome, Italy \\ ${ }^{17}$ INFN, Laboratori Nazionali di Frascati, Via Enrico Fermi 40, I-00044 Frascati, Italy \\ 18 Universität Siegen, Department of Physics, D-57068 Siegen, Germany \\ ${ }^{19}$ University of Udine, Department of Mathematics and Informatics, I-33100 Udine, Italy
}


It was the 15th of June of 2006 when the PAMELA satellite-borne experiment was launched from the Baikonur cosmodrome in Kazakstan. Then, for nearly ten years, PAMELA has been making high-precision measurements of the charged component of the cosmic radiation opening a new era of precision studies in cosmic rays and challenging our basic vision of the mechanisms of production, acceleration and propagation of cosmic rays in the galaxy and in the heliosphere. The study of the time dependence of the various components of the cosmic radiation from the unusual 23rd solar minimum through the maximum of solar cycle 24 clearly shows solar modulation effects as well as charge sign dependence. PAMELA measurement of the energy spectra during solar energetic particle events fills the existing energy gap between the highest energy particles measured in space and the ground-based domain. Finally, by sampling the particle radiation in different regions of the magnetosphere, PAMELA data provide a detailed study of the Earth $\mathrm{s}$ magnetosphere. In this highlight paper, PAMELA main results as well as recent progress about solar and heliospheric physics with PAMELA will be presented.

35th International Cosmic Ray Conference

10-20 July, 2017

Bexco, Busan, Korea

*Speaker. 


\section{Introduction}

The PAMELA (a Payload for Antimatter-Matter Exploration and Light-nuclei Astrophysics) satellite experiment was designed to study the charged component of the cosmic radiation, focusing on antiparticles. PAMELA was launched with a Soyuz-U rocket on June $15^{\text {th }}$ of 2006 from the Baikonur cosmodrome (Kazakhstan). The apparatus is hosted on the Russian Resurs-DK1 satellite, a commercial Earth-observation spacecraft. At first the orbit was elliptical (altitude varying between 355 and $584 \mathrm{~km}$ ) and semipolar (inclination of about $70^{\circ}$ ) and with a period of about 94 minutes. In 2010 the orbit was set to be circular with an almost fixed altitude of about $550 \mathrm{~km}$.

Initially planned for three years, PAMELA operation lasted until January 2016 when the data transmission from the satellite to the ground station in Moscow was interrupted because of technical problems with the satellite. The operation with the satellite and, consequently, with PAMELA were officially terminated sometime in 2016. The last PAMELA data were transmitted to ground on 24 January 2016.

The PAMELA experiment is the results of the collaboration between Italian (Universities and Istituto Nazionale di Fisica Nucleare I.N.F.N. Structures), German (Universität Siegen), Russian (Lebedev Physical Institute, Ioffe Physical Technical Institute, National Nuclear Research University MEPhI) and Swedish (KTH Royal Institute of Technology) institutes. Over the years, researchers from other institutes in Germany (Christian-Albrechts-Universität, Kiel), South Africa (North-West University, Potchefstroom) and USA (NASA Goddard Space Flight Center, New Mexico State University, University of New Hampshire) joined the PAMELA Collaboration on specific science topics ranging from solar physics to studies of solar modulation effects.

The PAMELA main scientific objectives were the measurement of the energy spectra and composition of the charged cosmic radiation from few tens of $\mathrm{MeV}$ up to $1 \mathrm{TeV}$ in kinetic energy. Specifically, the data allowed the study of the antiproton spectrum up to $350 \mathrm{GeV}$, of the positron spectrum up to $200 \mathrm{GeV}$, of the electron spectrum up to $600 \mathrm{GeV}$, of the proton and helium nuclei spectra up to 1.2 and $0.6 \mathrm{TeV} / \mathrm{n}$ respectively and of the nuclei spectra (from Li to O) up to $\sim 100 \mathrm{GeV} / \mathrm{n}$. Furthermore, new physics such as existence of antinuclei (with a $\overline{\mathrm{He}} / \mathrm{He}$ sensitivity of $10^{-7}$ ), of new forms of matter, e.g. strangelets, was studied as well as possible structures in cosmic ray (CR) spectra arising from e.g. dark matter or new astrophysical sources. Furthermore, PAMELA was well suited to conduct studies of CR acceleration and propagation mechanisms in the Galaxy, solar modulation effects, the emissions of Solar Energetic Particles (SEPs) inside the heliosphere and investigate the particles in the Earth's magnetosphere.

In the following, PAMELA main results as well as recent progress about solar and heliospheric physics obtained with PAMELA data will be presented.

\section{The PAMELA instrument}

The apparatus is shown in Figure 1 and comprises the following subdetectors (from top to bottom): a Time-of-Flight system (TOF), a magnetic spectrometer, an anticoincidence system, an electromagnetic imaging calorimeter, a shower tail catcher scintillator (S4) and a neutron detector.

The PAMELA detector is built around a permanent magnet that hosts the tracking system composed of six planes of double-sided silicon sensors, which form the magnetic spectrometer. 


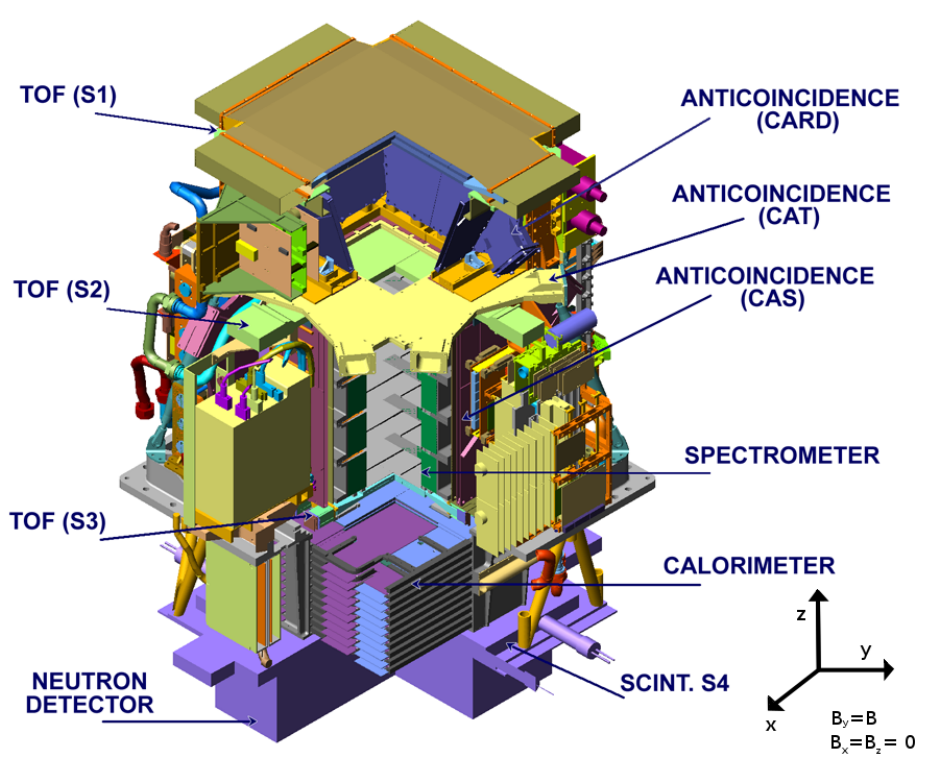

Figure 1: PAMELA and its sub-detectors.

The magnetic spectrometer measures the particle rigidity $r=p c / \mathrm{Ze}^{1}$ and the ionization energy losses $(\mathrm{dE} / \mathrm{dx})$. The rigidity measurement is done through the reconstruction of the trajectory based on the impact points on the tracking planes and the resulting determination of the curvature due to the Lorentz force. The Time-of-Flight (ToF) system comprises three double layers of plastic scintillator paddles with the first two placed above and the third immediately below the magnetic spectrometer. The ToF system provides the measurements of the particle velocity combining the time of passage information with the track length derived from the magnetic spectrometer. From the information on the particle incoming direction and the curvature in the spectrometer negatively charged particles are distinguished from positively charged particles.

The sampling imaging calorimeter has 16.3 radiation lengths ( 0.6 interaction lengths) and is the main sub-detector used for hadron-lepton separation. The topological and energetic information of the shower development in the calorimeter allows to reach a rejection power of proton against positron of the order of $10^{-4}$. The shower tail catcher and the neutron detector beneath provide additional information for the discrimination. An anticoincidence system is used to reject spurious events in the off-line phase.

More details about the apparatus can be found in [1].

\section{Review of PAMELA main scientific results}

Reviews of most PAMELA results have already been published [2, 3], here we will revise the most significant ones with a focus on their relevance for studies of solar and heliospheric physics.

Figure 2 shows the original PAMELA results on the galactic cosmic ray (GCR) positron fraction along with other previous measurements and a theoretical secondary prediction (see [4] and references within).

\footnotetext{
${ }^{1} \mathrm{p}$ and $\mathrm{Ze}$ are respectively the particle momentum and charge, and $\mathrm{c}$ the speed of light.
} 


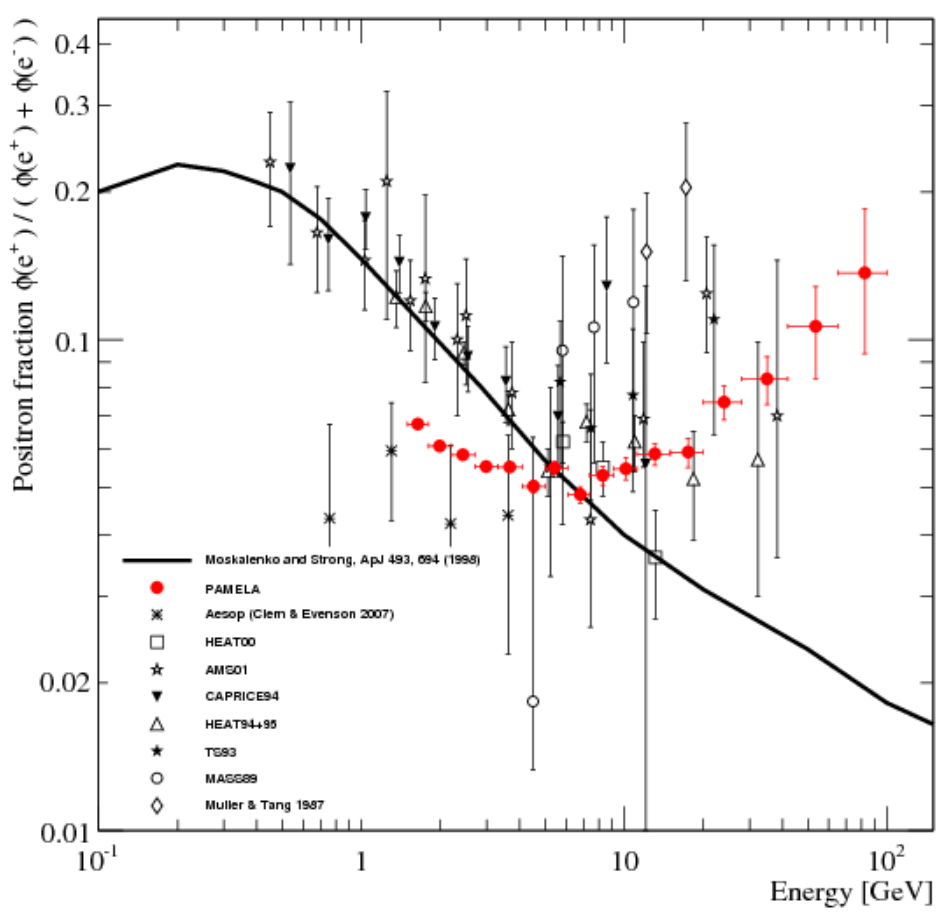

Figure 2: Galactic cosmic ray positron fraction measured by PAMELA [4], along with other previous measurements and a theoretical secondary prediction (see [4] and references within).

Undoubtedly the best known PAMELA result, these positron fraction data were extremely intriguing because of the differences with expectations for positrons produced by the inelastic interactions of CR nuclei with the interstellar medium at high energies but also for the differences at low energies respect to the majority of the older measurements. In subsequent years, the results were proven correct by the Fermi telescope [5] and, especially, by the AMS-02 magnetic spectrometer [6] as can be seen in Figure 3.

The significance of the high energy positron fraction PAMELA data have been extensively discussed elsewhere (e.g. [8]), here the differences at low energies will be addressed and explained as result of sign-charge dependence of the solar modulation.

Traversing the heliosphere, GCR are scattered by the irregularities of the turbulent heliospheric magnetic field (HMF) embedded into the solar wind and undergo convection and adiabatic deceleration in the expanding solar wind. As a consequence, the intensity of CR at Earth decreases with respect to the local interstellar spectrum [9]. Solar modulation has large effects on low energy CRs (less than a few $\mathrm{GeV}$ ), while the effects significantly decrease as the energies increase becoming negligible above a few tens of GeV. Moreover, because of the 11-year solar activity cycle, the intensity of CRs inside the heliosphere changes with time. This can be clearly seen in the energy spectra of cosmic rays. Figure 4 shows the all-electron (electrons plus positrons) spectrum measured by PAMELA along with other recent measurements. The difference with AMS-02 [14] below about 


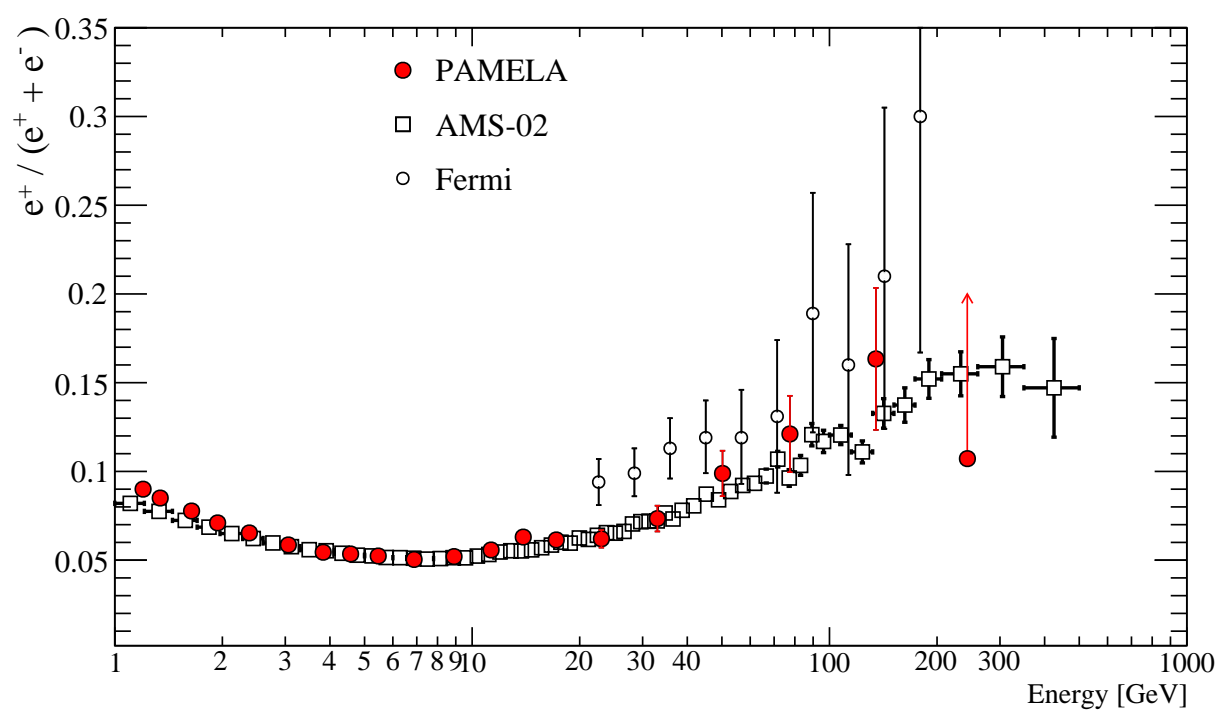

Figure 3: Galactic cosmic ray positron fraction measured by PAMELA [7], along with the recent Fermi [5] and AMS-02 [6] measurements. Only statistical errors are shown.

$10 \mathrm{GeV}$ respect to the excellent agreement at higher energies can be ascribed to solar modulation considering that PAMELA data refer to a period of solar minimum (July 2006-December 2009) and AMS-02 to a period of significantly higher solar activity (May 2011-November 2013).

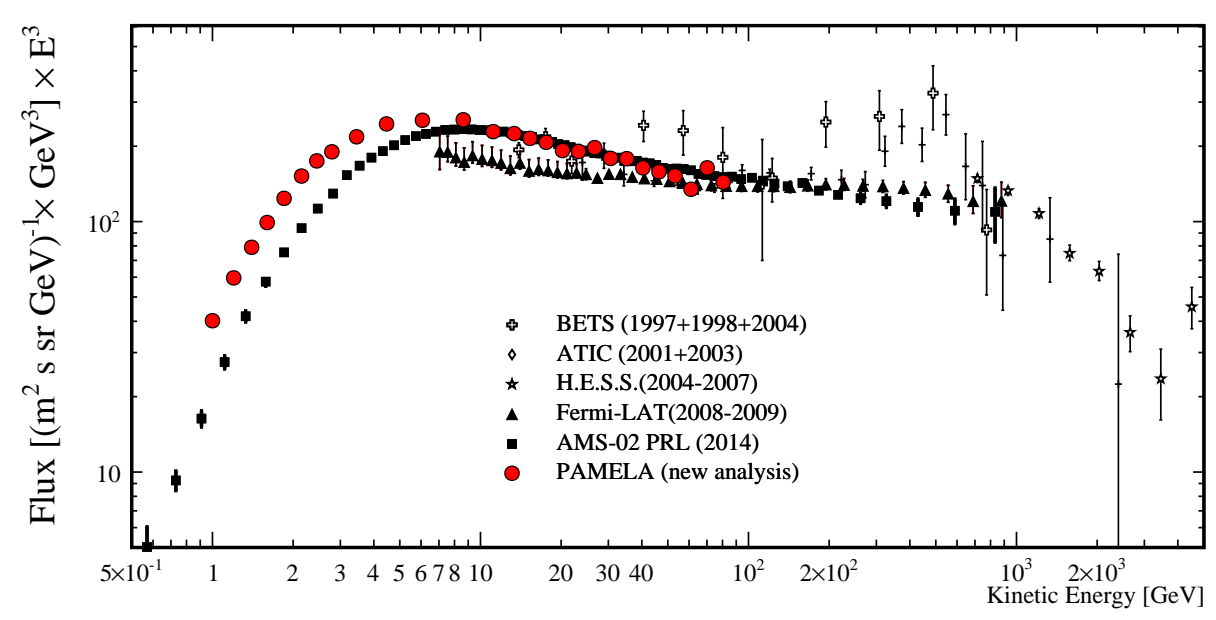

Figure 4: All electron energy spectrum measured by PAMELA [3] along with other recent results [10, 11, $12,13,14]$. Only statistical errors are shown.

A similar behavior, excellent agreement at high rigidities (above tens of GV) and differences at lower rigidities, is found also in the proton and helium nuclei spectra measured by PAMELA [15] and AMS-02 [16, 17], see Figure 5. Also in this case the differences at low rigidities can be ascribed 

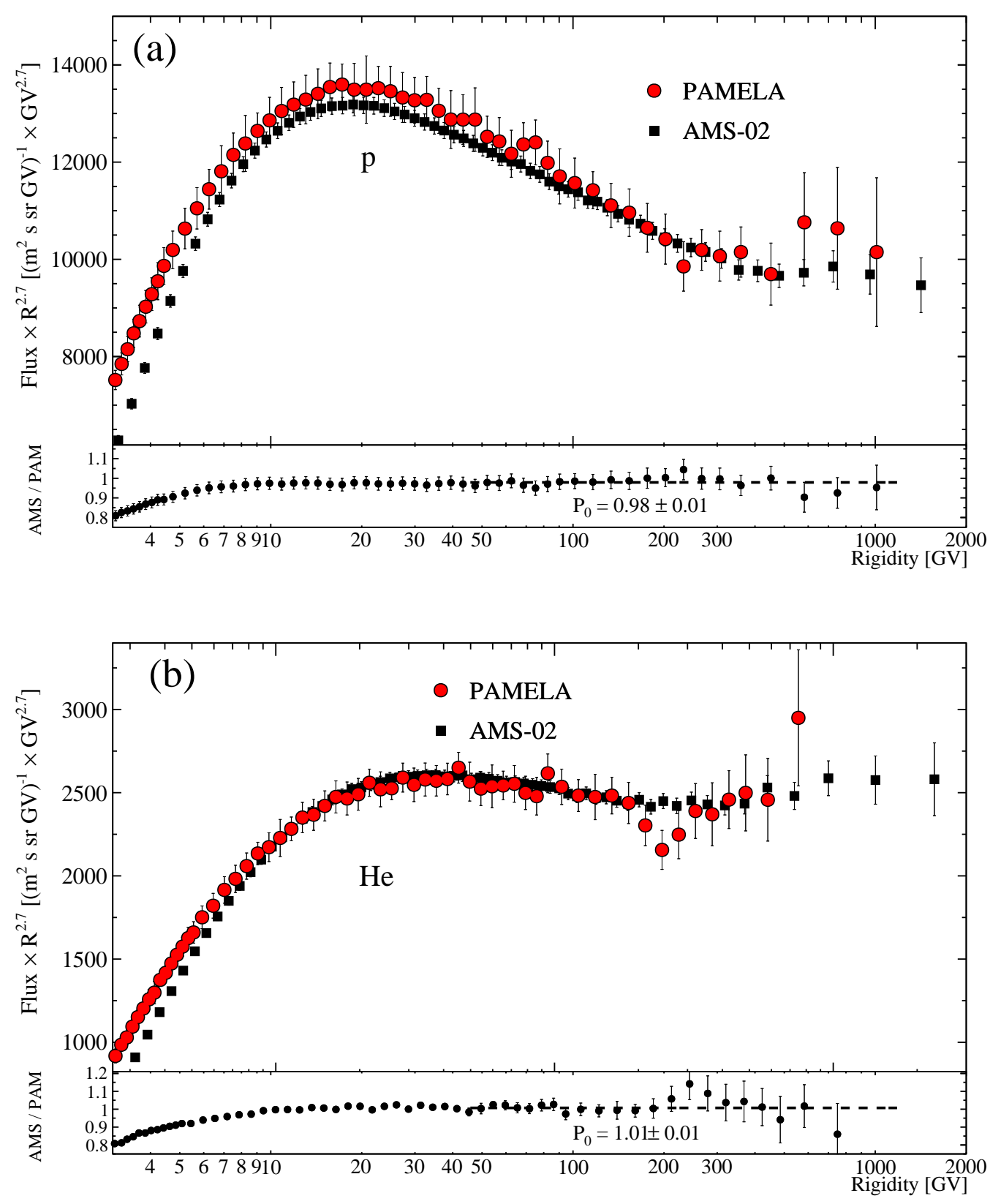

Figure 5: (a) Top panel: comparison of the proton fluxes measured by PAMELA [15] and AMS-02 [16]. Lower panel: AMS-02 and PAMELA proton flux ratio. The value of $\mathrm{P}_{0}$ results from a linear fit between $50 \mathrm{GV}$ and 1 TV. (b) Top panel: comparison of the helium fluxes measured by PAMELA [15] and AMS-02 [17]. Lower panel: AMS-02 and PAMELA He flux ratio. The value of $\mathrm{P}_{0}$ results from a linear fit between $50 \mathrm{GV}$ and $1 \mathrm{TV}$. 


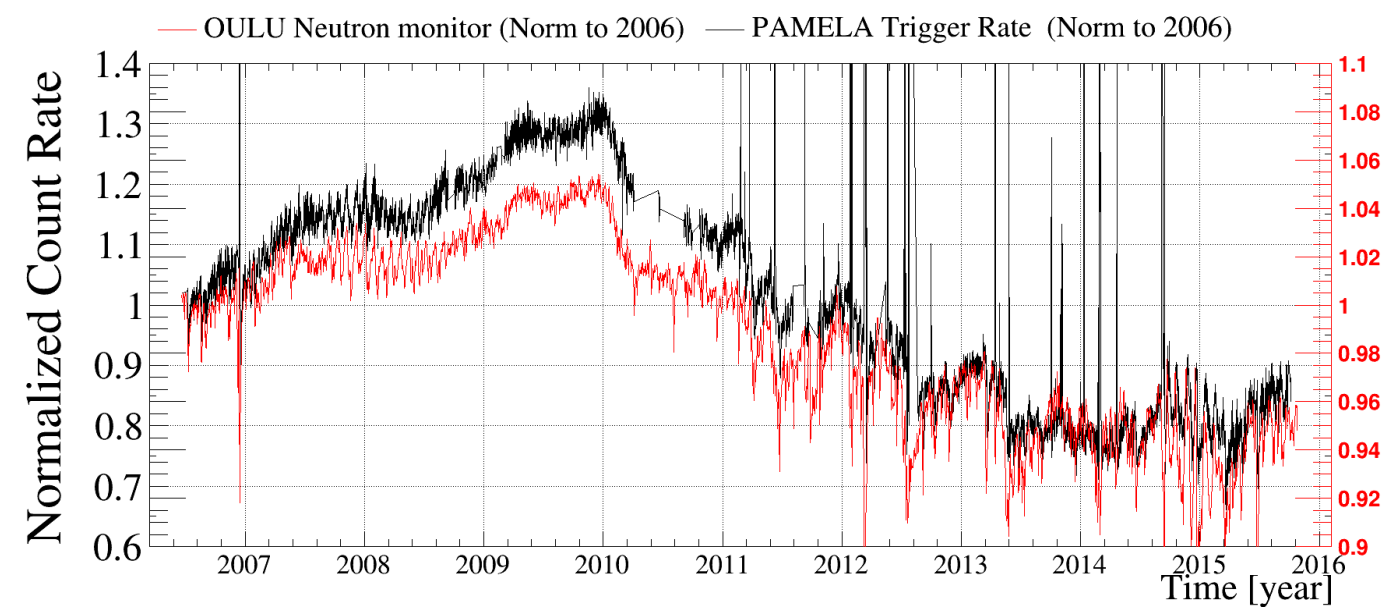

Figure 6: PAMELA trigger rate and Oulu neutron monitor count rate (data taken from http://cosmicrays.oulu.fi/) Data are normalized to July 2006.

to different period of solar activity: July 2006-Mar 2008 for PAMELA, May 2011-November 2013 for AMS-02.

Another conclusion that can be drawn from these comparisons is the striking agreement between various measurements, especially those of PAMELA and AMS-02, which is testament of the quality and reliability of these results and of the conclusion that can be drawn from them.

\section{Propagation in the heliosphere}

As previously discussed, because of the interaction with the solar wind the CR intensity measured at Earth decreases with respect to the local interstellar spectrum, i.e. the CR intensity as measured just outside the heliospheric boundary [18]. In addition, long-term changes in the solar activity, i.e. the 11-year solar cycle, produce time variations in the near-Earth CR intensity. This behavior is illustrated in Figure 6 that shows PAMELA trigger rate and neutron monitor counts (both normalized to mid 2006) measured by the Oulu neutron monitor ${ }^{2}$. The solar cycle can be clearly noticed in both sets of data: increasing counting rate from mid 2006 till late 2009 (solar minimum) followed by a sharp decrease with a leveling out in mid 2013 (solar maximum). The different relative increase over the solar minimum as well as the spikes (SEPs) noticeable nearly only in PAMELA trigger rate are due to the higher sensitivity to low energies $(<1 \mathrm{GeV})$ particles of PAMELA instrument respect to neutron monitors.

Precise measurements of the time-dependent CR spectra are essential to understand the cosmic ray propagation through the heliosphere. Furthermore, the experimental and theoretical investigation of this system provides information that can be easily applied to larger astrophysical systems. The possibility of performing in-situ measurements makes the interplanetary medium the ideal environment to test the theory of propagation of charged particles in magnetic fields under conditions

\footnotetext{
${ }^{2}$ Cosmic rays colliding with molecules in the atmosphere produce air showers of secondary particles including neutrons. The neutron monitor count rate is thus proportional to the intensities of the CR flux at Earth.
} 
which approximate typical cosmic condition. Hence, very useful information for understanding the origin and propagation of cosmic rays in the Galaxy can be derived.

Figure 7 shows the proton intensity at different rigidities (normalized to July 2006) measured by PAMELA from the beginning of the data-taking, in July 2006, until September 2014 [19]. More than $2 \times 10^{8}$ protons were collected. The high statistic allowed to sample the proton fluxes over a Carrington Rotation (period of 27.27 days). Holes in the presented data are due to periods of

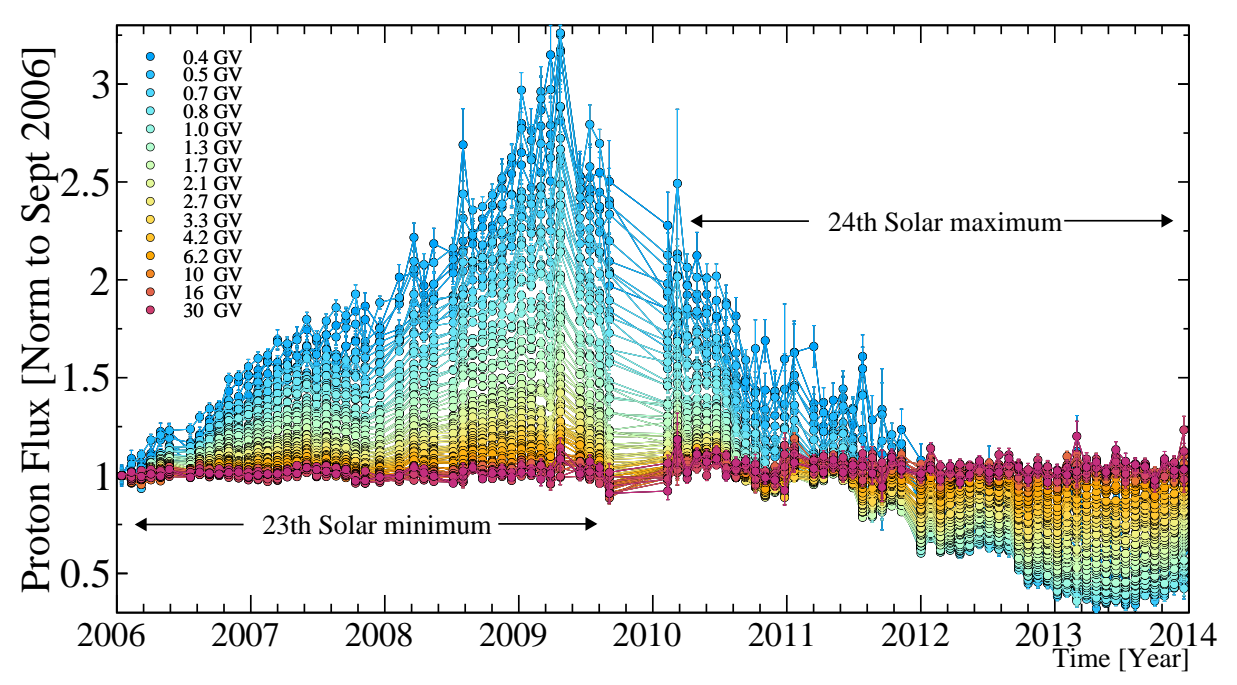

Figure 7: Time-dependent proton intensities (normalized to July 2006) measured by PAMELA between July 2006 and May 2014. Each point represents a $\sim 27$ days time period. Holes are due to missing data from satellite or to the off-line exclusion of sudden transient from the Sun as solar flares.

non operation of either the PAMELA instrumentation or the satellite. Furthermore, time periods during solar events were excluded from the analysis. The major modulation effects are experienced at the lowest rigidity $(0.4 \mathrm{GV})$ with an increase of about a factor 3 with respect to July 2006. At higher rigidities the solar modulation effects decrease and above $30 \mathrm{GV}$ the proton flux is time-independent within the experimental uncertainties. The 23rd solar minimum activity and the consequent minimum modulation conditions for CRs were unusual (e.g. see [18]). It was expected that the 24th solar cycle would begin in early 2008. Instead solar minimum modulation conditions continued until the end of 2009 when PAMELA measured the highest cosmic ray proton spectrum from the beginning of the space age. From 2010, as the solar activity started to increase, the proton intensity showed a decrease up to the beginning of 2013, when the maximum activity of the 24th solar cycle was reached. After mid 2014, the lowest energy protons showed again an increasing trend as a consequence of the solar activity decrease.

As showed in Figure 5, above $50 \mathrm{GV}$ the agreement between PAMELA and AMS-02 proton fluxes is within $2 \%$. However, below this rigidity, the measurements differs because of solar modulation effects. In order to compare the proton fluxes below $50 \mathrm{GV}$, a new analysis was performed with the PAMELA data collected between May 2011 and November 2013, i.e. the time period corresponding to AMS-02 published spectrum. Figure 8 shows this new proton spectrum compared with the AMS-02 results. Now, an excellent agreement can be noticed also at low rigidities (e.g., 


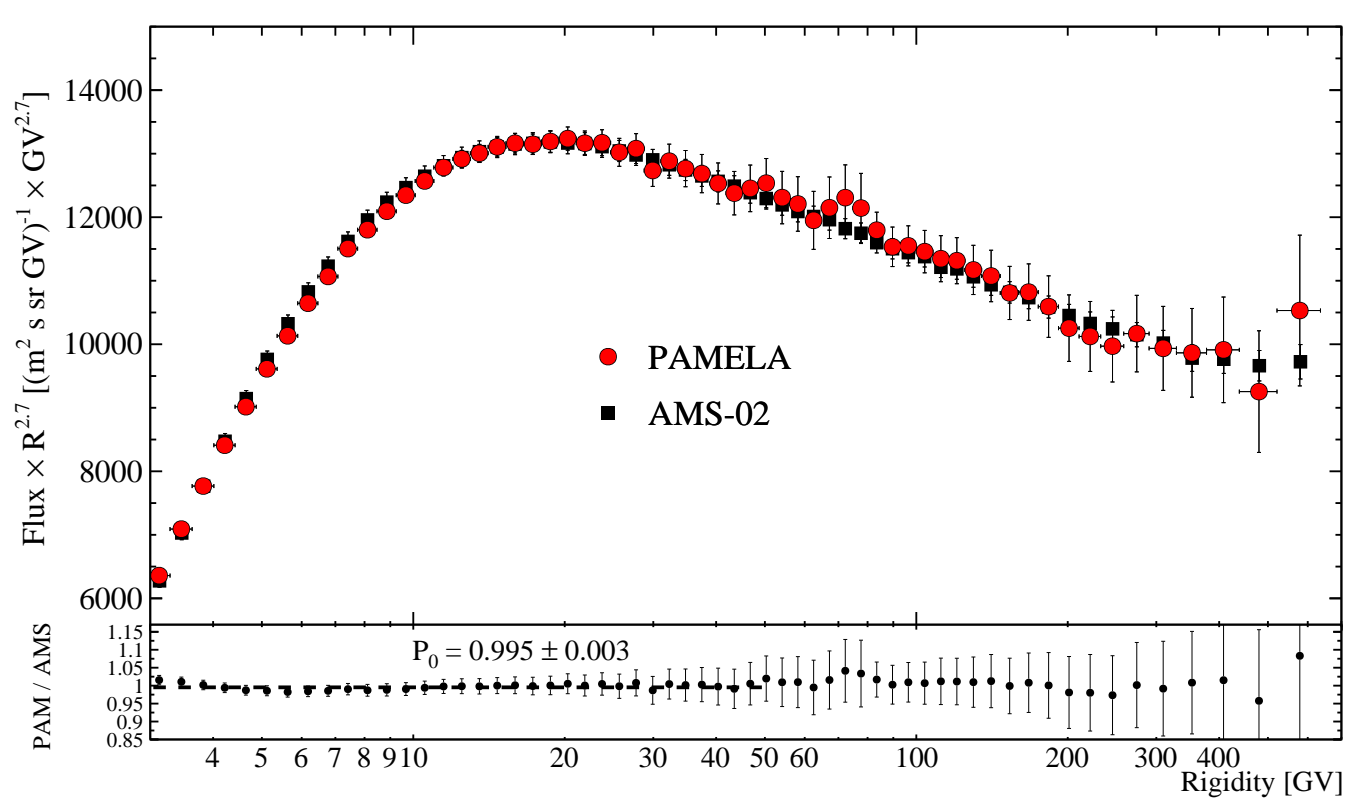

Figure 8: The PAMELA proton flux evaluated between May 2011 and November 2013 compared with recent AMS-02 measurement [16]. Only statistical errors are showed. The agreement between PAMELA and AMS-02 is excellent along the whole energy range. $\mathrm{P}_{0}$ results from a constant fit on the flux ratio between 3 and $50 \mathrm{GV}$.

see $\mathrm{P}_{0}$ resulting from a constant fit to the flux ratio between 3 and $50 \mathrm{GV}$ ).

On top of the time dependence, a charge sign dependence of the solar modulation is expected. The gradients and curvatures present in the HMF induce drift motions that depend on the particle charge sign (e.g. see [20]). During so-called $\mathrm{A}<0^{3}$ polarity cycles such as solar cycle 23 , when the heliospheric magnetic field is directed toward the Sun in the northern hemisphere, negatively charge particles undergo drift motion mainly from the polar to the equatorial regions and outwards along the heliospheric current sheet. Positively charged particles drift mainly in opposite directions. The situation reverses when the solar magnetic field changes its polarity at each solar maximum. Hence, the charge-sign dependence introduces a 22-years cycle.

Strong evidence of charge-sign dependent solar modulation was provided by PAMELA measurements of the positron to electron ratio, performed between July 2006 and December 2015 [21, 22]. These data, shown in Figure 9, present the first clear indication of the evolution of drift effects during different phases of the solar activity and the dependence on particle rigidity. Data were normalized to the values measured between July and December 2006. The red shaded area represents the time interval during which the process of polar field reversal took place [23].

The results show a time dependence of the positron to electron ratio. In the first two energy intervals of Figure $9(0.5-1$ and $1-2.5 \mathrm{GeV})$ an increase of the ratio was observed up to the end of 2009. During this time period positrons at Earth increased about 20\% more than electrons. For

\footnotetext{
${ }^{3}$ In the complex sun magnetic field the dipole term nearly always dominates the magnetic field of the solar wind. A is defined as the projection of this dipole on the solar rotation axis.
} 

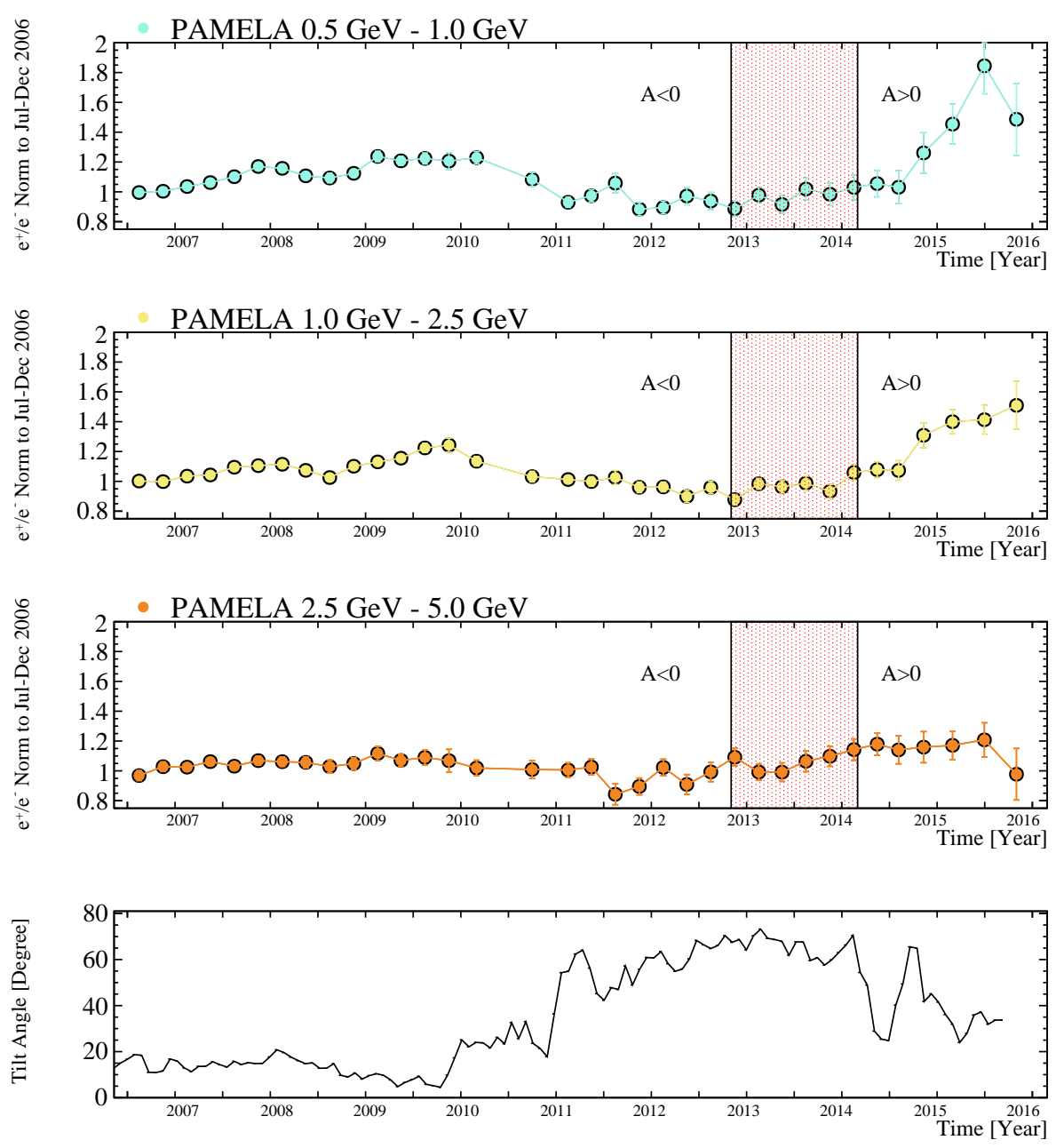

Figure 9: The positron to electron ratios normalized to July - December 2006 measured at Earth by the PAMELA experiment for three different energy intervals. The colored lines provide connection among the points to guide the eye. The shaded area corresponds to the period with no well defined HMF polarity [23]. Bottom panel: the tilt angle as a function of time.

the third energy interval $(2.5-5.0 \mathrm{GeV})$ this increase was $\sim 10 \%$.

Until the middle of 2013 the ratio remained constant and slowly increased up to the middle of 2014 when a sudden rise was observed up to late 2015 for the first two panels of Figure 9 where positrons increased respectively about $80 \%$ and $50 \%$ more than electrons. This sudden rise is not observed for the highest energy interval, where the positrons increased only about $20 \%$ more than electrons. The sudden rise measured during this period appears to be a consequence of the polarity reversal of the HMF.

The trends in the observational data shown in Figure 9 can be interpreted in terms of particle drifts. For the period 2006 to 2009 , the tilt angle ${ }^{4}$ decreased slowly to reach a minimum value at

\footnotetext{
${ }^{4}$ In the context of this charge-sign dependent modulation, the tilt angle of the wavy heliospheric current sheet is the most appropriate proxy for solar activity.
} 


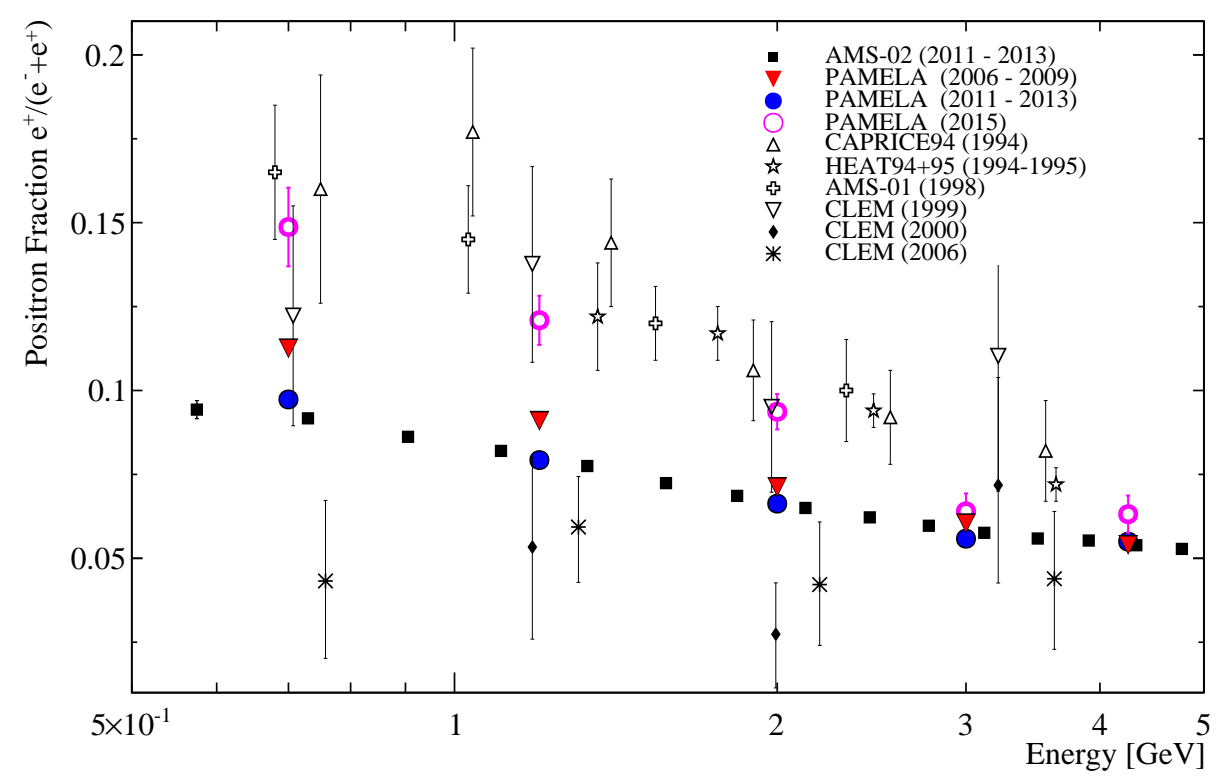

Figure 10: The PAMELA positron fraction for three time periods: July 2006-December 2009, (solar minimum, as in [7]), May 2011-November 2013 (as AMS-02 results [6]), January-December 2015, along with other recent measurements: HEAT94+95 [24], CAPRICE94 [25], AMS-01 [26], Aesop [27], AMS-02 [6]. The results from $[24,25,26]$ refer to the previous $\mathrm{A}>0$ solar cycle.

the end of 2009 as shown in the bottom panel of Figure 9. During this $A<0$ magnetic polarity cycle, positrons drifted towards the Earth mainly through the equatorial regions of the heliosphere, encountering the changing wavy current sheet, while electrons drifted inwards mainly through the polar regions of the heliosphere and were consequently less influenced by the current sheet. The positron flux therefore increased relatively more than the electron flux with a decreasing tilt angle until the end of 2009. From 2010 onwards, the tilt angle increased sharply so that the positron flux also decreased proportionally faster than the electron flux and the ratio $e^{+} / e^{-}$decreased. This continued until increased solar activity influenced both fluxes equally and the ratio $e^{+} / e^{-}$became steady. From the end of 2012, the solar magnetic field had gone into a reversal phase, which lasted until the beginning of 2014. After this turbulent reversal phase (from $A<0$ to $A>0$ ) the positrons gradually started to drift inwards through the polar regions of the heliosphere to the Earth while the electrons started to drift inwards through the equatorial regions so that the positron flux increased proportionally more than for electrons.

The charge-sign dependence introduced by drift motion is also visible in Figure 10 were the positron fractions measured by PAMELA in various time periods and by other experiments are shown. A good agreement can be noticed between PAMELA and AMS-02 results taken over the same time period. Moreover, the positron fraction measured in 2015 draws near to the measurements $[24,25,26]$ from the previous A $>0$ solar cycle in the 90's.

PAMELA data are currently under analysis to study the temporal variation of additional particle species, focusing in particular on the solar modulation of GCR helium nuclei fluxes. 


\subsection{Solar Particle Events}

In addition to modulate the GCR energy spectra, the Sun contributes to the particle intensities in the heliosphere emitting a population of particles with energy ranging from a few tens of $\mathrm{keV}$ to a few $\mathrm{GeV}$. These solar energetic particles are associated with solar flares and coronal mass ejection (CME). These events inject large amounts of nuclei into space, whose composition varies from event to event and it is heavily linked to the production mechanisms that take place. Whether the Sun accelerates particles at low altitudes through magnetic reconnection or higher in the outermost layers of its atmosphere (like the corona) through coronal mass ejection-driven shocks, or perhaps an admixture of the two, is still unclear [28, 29]. This kind of uncertainty involves both low energy particles measured in situ and the higher energy populations which lead to particularly energetic phenomena called Ground Level Enhancements. These are produced when solar protons in the $\sim \mathrm{GeV}$ range start a nuclear cascade through the Earth's atmosphere that can be observed by detectors at ground level, such as neutron monitors, as an increase above the background produced by ordinary galactic cosmic rays [30].

PAMELA fills the largely unexplored energy gap between the particles detected in space (below few hundreds of $\mathrm{MeV}$ ) and particles detected at ground level (above few $\mathrm{GeV}$ ). First PAMELA observation of SEPs occurred in late 2006 with the December $13^{\text {th }}$ and $14^{\text {th }}$ events which also represent the first direct measurement of SEPs in space with a single instrument in the energy range from $\sim 80 \mathrm{MeV} / \mathrm{n}$ to $\sim 3 \mathrm{GeV} / \mathrm{n}$. Solar helium nuclei (up to $1 \mathrm{GeV} / \mathrm{n}$ ) and protons (up to $\sim 2 \mathrm{GeV} / \mathrm{n}$ ) were recorded [31]. Since then, several other SEP events were measured by PAMELA [32]. Table 1 reports the list of the 28 major SEP events observed by PAMELA between 2006 July and 2014 September. For each event, the class/location information about the source flare are displayed, along with the related CME speed and width. All events were associated with $X$ - and $M$-class flares except for the 2013 September 30 ( $C$-class), and with full halo CMEs except for the 2011 September 06, the 2012 July 08 and the 2013 October 28 events (partial halo CMEs).

\subsection{Short-term variation in the galactic cosmic ray intensity}

On top of the long-term solar modulation, short-term modulation effects also occur. For example, the near Earth GCR intensity is greatly modified by transient phenomena in the solar environment. In particular, interplanetary transients such as CMEs can induce Forbush decreases, i.e. sudden suppression of GCR intensity near the Earth [33]. Such phenomena can last up to several days and suppress the GCR intensity measured during quite Sun condition of about $30 \%-40 \%$.

Recurrent short-term GCR decreases have also been measured in association with the passage of Co-rotating Interaction Regions (CIRs). Such regions of compressed plasma, formed at the leading edges of high-speed solar wind streams originating from coronal holes and interacting with the preceding slow solar wind, are a well known cause of periodic CR decreases [34, 35].

In addition to the SEP event observed by PAMELA on December 13 2006, a significant Forbush decrease was observed when the full-halo CME produced by the solar event reached and passed Earth. PAMELA instrument measured the effect of this transient in the GCR intensity in the rigidity range from $400 \mathrm{MV}$ to $20 \mathrm{GV}$. The rigidity reconstruction accuracy and the high collected statistics allowed to perform a rigidity dependent study of the Forbush decrease for various CR components: protons, helium nuclei and electrons [36]. The helium and proton intensities show a 


\begin{tabular}{|c|c|c|c|c|c|}
\hline \multicolumn{2}{|r|}{ SEP Event } & \multicolumn{2}{|c|}{ Flare } & \multicolumn{2}{|c|}{ CME } \\
\hline \# & Date & Class & Location & Speed & Width \\
\hline 1 & 2006 Dec 13 & X3.4 & S06W23 & 1774 & $\mathrm{H}$ \\
\hline 2 & 2006 Dec 14 & X1.5 & S06W46 & 1042 & $\mathrm{H}$ \\
\hline 3 & 2011 Mar 21 & N.A. & $>$ W90 & 1341 & $\mathrm{H}$ \\
\hline 4 & 2011 Jun 07 & M2.5 & S21W54 & 1255 & $\mathrm{H}$ \\
\hline 5 & 2011 Sep 06 & M5.3 & N.A. & N.A. & N.A. \\
\hline 6 & 2011 Sep 07 & $\mathrm{X} 2.1$ & N.A. & N.A. & N.A. \\
\hline 7 & 2011 Nov 04 & N.A. & N.A. & N.A. & N.A. \\
\hline 8 & 2012 Jan 23 & M8.7 & N28W21 & 2175 & $\mathrm{H}$ \\
\hline 9 & 2012 Jan 27 & X1.7 & N27W71 & 2508 & $\mathrm{H}$ \\
\hline 10 & 2012 Mar 07 & X5.4 & N17E27 & 2684 & $\mathrm{H}$ \\
\hline 11 & 2012 Mar 13 & M7.9 & N17W66 & 1884 & $\mathrm{H}$ \\
\hline 12 & 2012 May 17 & M5.1 & N11W76 & 1582 & $\mathrm{H}$ \\
\hline 13 & $2012 \mathrm{Jul} 06$ & X1.1 & S13W59 & 1828 & $\mathrm{H}$ \\
\hline 14 & $2012 \mathrm{Jul} 08$ & M6.9 & S17W74 & 1497 & 157 \\
\hline 15 & 2012 Jul 12 & X1.4 & N.A. & N.A. & N.A. \\
\hline 16 & 2012 Jul 19 & M7.7 & S13W88 & 1631 & $\mathrm{H}$ \\
\hline 17 & 2012 Jul 23 & N.A. & $>$ W90 & 2003 & $\mathrm{H}$ \\
\hline 18 & 2013 Apr 11 & M6.5 & N09E12 & 861 & $\mathrm{H}$ \\
\hline 19 & 2013 May 22 & M5.0 & N13W75 & 1466 & $\mathrm{H}$ \\
\hline 20 & 2013 Sep 30 & C1.3 & N17W29 & 1179 & $\mathrm{H}$ \\
\hline 21 & 2013 Oct 28 & M5.1 & N.A. & N.A. & N.A. \\
\hline 22 & 2013 Nov 02 & N.A. & N.A. & N.A. & N.A. \\
\hline 23 & 2014 Jan 06 & N.A. & $>$ W90 & 1402 & $\mathrm{H}$ \\
\hline 24 & 2014 Jan 07 & $\mathrm{X} 1.2$ & S15W11 & 1830 & $\mathrm{H}$ \\
\hline 25 & 2014 Feb 25 & $\mathrm{X} 4.9$ & S12E82 & 2145 & $\mathrm{H}$ \\
\hline 26 & 2014 Apr 18 & M7.3 & S20W34 & 1203 & $\mathrm{H}$ \\
\hline 27 & 2014 Sep 01 & N.A. & >W90 & N.A. & N.A. \\
\hline 28 & 2014 Sep 10 & X1.6 & N14E02 & 1267 & $\mathrm{H}$ \\
\hline
\end{tabular}

Table 1: List of the major SEP events observed by PAMELA between 2006 July and 2014 September. For each event, the class/location information about the source flare are displayed, along with the related CME speed $(\mathrm{km} / \mathrm{s})$ and width (deg, or " $\mathrm{H}$ " in case of full halo CMEs). Flare/CME data are from https://cdaw.gsfc.nasa.gov/CME_list/sepe/.

good agreement for both the amplitude and the recovery time. On the contrary electrons show on average a faster recovery time with respect to protons and helium nuclei with a similar amplitude. This effect could be interpreted as a charge sign dependence introduced by drift motions that affect the GCRs during their propagation through the heliosphere during a negative polarity cycle. In addition to the temporal variation produced by the CME propagation in heliosphere, a periodicity of about 13 days was observed in the proton and helium flux after the Forbush decrease. This periodicity lasted for about two months and had a mean amplitude of about $7 \%$. This temporal variation was compared with the the temporal evolution of the solar wind (SW) parameters like the SW velocity, the proton density, the HMF intensity and others. A correlation was found between the GCR temporal variation and some specific SW features who could point to the passage of prominent structures of compressed plasma in the heliosphere like CIR. However, since the he- 
liospheric current sheet (HCS) is often crossed in the slow solar wind preceding a CIR, and may be incorporated into the CIR along with the slow solar wind, a correlation between this periodic intensity variation and the HCS crossing cannot be excluded.

Finally, a distinct feature was found in the low energy ( $80 \mathrm{MeV}-10 \mathrm{GeV}$ ) proton fluxes measured by PAMELA with a peculiar period of $\sim 400$ days. This effect was also reported by the worldwide network of Neutron Monitors but, to our knowledge, it is the first time that it has been observed in space at this low energies. This effect could be linked to the turbulent environment of the heliosphere. However, it is worth noticing that the larger proton fluxes were observed when the Earth intersected the IMF lines coming from Jupiter hinting to a possible, surprising, contribution from the intense Jupiter magnetosphere [37].

\section{Conclusions and acknowledgments}

It was the 15th of June of 2006 when the PAMELA satellite-borne experiment was launched from the Baikonur cosmodrome in Kazakstan. Then, for nearly ten years, PAMELA has been making high-precision measurements of the charged component of the cosmic radiation opening a new era of precision studies in cosmic rays and challenging our basic vision of the mechanisms of production, acceleration and propagation of cosmic rays in the galaxy and in the heliosphere. In addition to the results discussed in this paper, at the conference the PAMELA Collaboration presented results on lithium and beryllium isotopes [38], on solar modulation of GCR deuterons [39] and on effects due to the Earth's magnetosphere [40].

We would like to thank E. C. Christian, G. A. de Nolfo, M. S. Potgieter, J. M. Ryan and S. Stochaj for fruitful collaboration on the study of solar physics and solar modulation. We acknowledge partial financial support from The Italian Space Agency (ASI) under the program "Programma PAMELA - attivitá scientifica di analisi dati in fase E". We also acknowledge support from Deutsches fur Luft- und Raumfahrt (DLR), The Swedish National Space Board, The Swedish Research Council, The Russian Space Agency (Roscosmos) and Russian Science Foundation.

\section{References}

[1] P. Picozza et al., Astropart. Phys. 27 (2007) 296

[2] O. Adriani et al., Phys. Rept. 544 (2014) 323

[3] O. Adriani et al., to appear in Nuovo Cimento (2017)

[4] O. Adriani et al., Nature 458 (2009) 607

[5] M. Ackermann et al., Phys. Rev. Lett. 108 (2012) 011103

[6] L. Accardo et al., Phys. Rev. Lett. 113 (2014) 121101.

[7] O. Adriani et al., Phys. Rev. Lett. 111 (2013) 081102

[8] M. Boezio et al., New J. Phys. 11 (2009) 105023

[9] M. S. Potgieter, Living Reviews in Solar Physics 10 (2013) 3

[10] S. Torii et al., Astrophys. J. 559 (2001) 973

[11] J. Chang et al., Nature 456 (2008) 362 
[12] F. Aharonian et al., Phys. Rev. Lett. 101 (2008) 261104

[13] A. A. Abdo et al., Phys. Rev. Lett. 102 (2009) 181101

[14] M. Aguilar it et al., Phys. Rev. Lett. 113 (2014) 221102

[15] O. Adriani et al., Science 332 (2011) 69

[16] M. Aguilar et al., Phys. Rev. Lett. 114 (2015) 171103

[17] M. Aguilar et al., Phys. Rev. Lett. 115 (2015) no.21, 211101

[18] M. S. Potgieter, Brazilian Journal of Physics 442014581

[19] B. Panico et al., "Time dependence of the proton and helium flux measured by PAMELA", this conference (2017)

[20] S. E. S. Ferreira and M. S. Potgieter, Astrophys. J.. 603 (2004) 744

[21] O. Adriani et al., Phys. Rev. Lett. 116 (2016) 241105

[22] R. Munini et al., "Ten years of positron and electron solar modulation measured by the PAMELA experiment.", this conference (2017)

[23] X. Sun and J. T. Hoeksema and Y. Liu and J. Zhao, Astrophys. J. 798 (2015) 114

[24] S. W. Barwick et al., Astrophys. J. 482 (1997) L191

[25] M. Boezio et al., Astrophys. J. 532 (2000) 653

[26] J. Alcaraz et al., Phys. Lett. B 484 (2000) 10

[27] J. Clem and P. Evenson, Proc. 30th Intl Cosmic Ray Conf., Merida (2007).

[28] T. Mathews and D. Venkatesan, Nature 345 (1990) 600

[29] G. A. Bazilevskaya, Journal of Physics: Conference Series 7982017012034

[30] D. V. Reames, Space Science Reviews 175 (2013) 53

[31] O. Adriani et al., Astrophys. J. 742 (2011) 102

[32] M. Mergé et al., "PAMELA measurements of solar energetic particle spectra", this conference (2017)

[33] H. V. Cane, Space Science Reviews 93 (200) 55

[34] C. W. Barnes and J. A. Simpson, Astrophys. J. 207 (1976) 91

[35] I. G. Richardson, Space Science Reviews 111 (2004) 267

[36] R. Munini et al., "Short-term variation in the galactic cosmic ray intensity measured with the PAMELA experiment", this conference (2017)

[37] M. Ricci et al., "Effect of the Jupiter magnetosphere on the Cosmic Ray protons measured with the PAMELA space experiment", this conference (2017)

[38] W. Menn et al., "Cosmic-Ray Lithium and Beryllium Isotopes in the PAMELA-Experiment", this conference (2017)

[39] S. Koldobskiy et al., "Solar modulation of the galactic deuteron spectrum measured with the PAMELA experiment”, this conference (2017)

[40] V. Mikhailov et al., "Measurements of electron and positron fluxes below the geomagnetic cutoff by the PAMELA magnetic spectrometer", this conference (2017) 